Pacific Journal of Mathematics

BASES OF TENSOR PRODUCTS OF BANACH SPACES 


\section{BASES OF TENSOR PRODUCTS OF BANACH SPACES}

\section{B. R. Gelbaum and J. Gil de Lamadrid}

1. Introduction. In this note we use the conventions and notations of Schatten [4] with the exception that we use $B^{\prime}$ to indicate the dual (conjugate) space of a Banach space $B$ and $\left\langle x, x^{\prime}\right\rangle$ as the action of an element $x$ and a functional $x^{\prime}$ on each other. Schatten defines the tensor product $B_{1} \otimes{ }_{\alpha} B_{2}$ as the completion of the algebraic tensor product $B_{1} \otimes B_{2}$ of two Banach spaces $B_{1}$ and $B_{2}$, on which the cross norm $\alpha$ has been imposed. We discuss the proposition, "If $B_{1}$ and $B_{2}$ have Schauder bases, then $B_{1} \otimes_{\alpha} B_{2}$ has a Schauder basis." We prove this for $\alpha=\gamma$ $\left(B_{1} \otimes_{\gamma} B_{2}\right.$ is the trace class of transformations of $B_{1}^{\prime}$ into $B_{2}$ ). We also prove it for $\alpha=\lambda\left(B_{1} \otimes_{\lambda} B_{2}\right.$ is the class of all completely continuous linear transformations of $B_{1}^{\prime}$ into $B_{2}$ ) in the case in which the bases of $B_{1}$ and $B_{2}$ satisfy an "isometry condition". This condition is not very restrictive. We know of no instance in which it is not satisfied. Next we show that unconditional bases of $B_{1}$ and $B_{2}$ do not necessarily yield an unconditional basis for the tensor product, even in the nicest conceivable infinite dimensional case, that in which $B_{1}=B_{2}=$ Hilbert space, and the bases are orthonormal and identical.

We recall certain facts about Schauder bases, and set some general notation that we use throughout the paper. We usually work with a biorthogonal set $\Omega=\left\{x_{i}, x_{i}^{\prime}\right\}_{i}$ associated with a Banach space $B$, so that $\chi=\left\{x_{i}\right\}_{i}$ is a basis for $B$ with coefficients supplied by the corresponding sequence of functionals $\chi^{\prime}=\left\{x_{2}^{\prime}\right\}_{i}$. We will have to do with the closed linear manifold $B^{o}$ of $B^{\prime}$ generated by the elements of $\chi^{\prime}$. Since $B$ and $B^{\Omega}$ are in duality it is possible to embed $B$ in $\left(B^{\Omega}\right)^{\prime}$ by the same formula that effects the embedding of $B$ in $B^{\prime \prime}$. We denote by ${ }_{n} P_{m}$ the projection of $B$ defined by ${ }_{n} P_{m} x=\sum_{i=n}^{m}\left\langle x, x_{\imath}^{\prime}\right\rangle x_{i}$. The double sequence $\left.{ }_{n} P_{m}\right\}_{n, m}$ is uniformly bounded. We denote by $T^{\prime}$ the transpose of any transformation $T$. The following lemma, given without proof, is but a trivial strengthening of $[2, \mathrm{p} .18$, Theorem 1$]$.

LEMMA 1. Let $E$ be a dense vector subspace of $B, \Omega$ a biorthogonal set of $B$ such that $\chi \subset E$, the vector space spanned by $\chi$ is dense in $E$ and the sequence $\left\{{ }_{n} P_{m}\right\}_{n, m}$ is uniformly bounded on $E$. Then $\Omega$ defines $a$ basis for $B$.

2. The tensor product of two biorthogonal sets. Let $\Omega_{1}=\left\{x_{i}, x_{i}^{\prime}\right\}_{i}$ be a biorthogonal set of $B_{1}$ and $\Omega_{2}=\left\{y_{i}, y_{i}^{\prime}\right\}_{i}$ a biorthogonal set of $B_{2}$.

Received November 15, 1960. This research was supported in part by NSF research grant No. NSFG 14137 and in part by NSF research grant No. NSFG 11048. 
The elements $x_{i}^{\prime} \otimes y_{j}^{\prime}$ can be considered as belonging to $\left(B_{1} \otimes{ }_{\alpha} B_{2}\right)^{\prime}$ for any cross norm $\alpha$ [4 p. 43], and $\left\{x_{i} \otimes y_{j}, x_{i}^{\prime} \otimes y_{j}^{\prime}\right\}_{i, j}$ is clearly a biorthogonal set. We enumerate it, not by the diagonal method, i.e., as in the usual proof that the rationals are denumerable, but as follows: In the table

$$
\begin{array}{lll}
x_{1} \otimes y_{1} & x_{1} \otimes y_{2} & x_{1} \otimes y_{3} \ldots \ldots \\
x_{2} \otimes y_{1} & x_{2} \otimes y_{2} & x_{2} \otimes y_{3} \ldots . \\
x_{3} \otimes y_{1} & x_{3} \otimes y_{2} & x_{3} \otimes y_{3} \ldots .
\end{array}
$$

we simply order the elements by listing the entries on the two inner sides of each successive upper left hand block to obtain $x_{1} \otimes y_{1}, x_{1} \otimes y_{2}$, $x_{2} \otimes y_{2}, x_{2} \otimes y_{1}, x_{1} \otimes y_{3}, x_{2} \otimes y_{3}, x_{3} \otimes y_{3}, x_{3} \otimes y_{2}, x_{3} \otimes y_{1}, \cdots, x_{1} \otimes y_{k}, x_{2} \otimes$ $y_{k} \cdots x_{k} \otimes y_{k}, x_{k} \otimes y_{k-1}, \cdots, x_{k} \otimes y_{2}, x_{k} \otimes y_{1}, \cdots$. This double sequence with the given order is called the tensor product of $\chi_{1}=\left\{x_{i}\right\}_{i}$ and $\chi_{2}=$ $\left\{y_{j}\right\}_{j}$ and is denoted by $\chi_{1} \otimes \chi_{2}$. Similarly $\chi_{1}^{\prime} \otimes \chi_{2}^{\prime}$ denotes the set $\left\{x_{i}^{\prime} \otimes y_{j}^{\prime}\right\}_{i, j}$ with the corresponding order. The biorthogonal set formed by $\chi_{1} \otimes \chi_{2}$ and $\chi_{1}^{\prime} \otimes \chi_{2}^{\prime}$ is called the tensor product of $\Omega_{1}$ and $\Omega_{2}$ and denoted by $\Omega_{1} \otimes \Omega_{2}$.

THeOREM 1. If $\Omega_{1}$ defines a basis for $B_{1}$ and $\Omega_{2}$ defines a basis for $B_{2}$, then $\Omega_{1} \otimes \Omega_{2}$ defines a basis for $B_{1} \otimes_{\gamma} B_{2}$.

Proof. We show that the vector space spanned by $\chi_{1} \otimes \chi_{2}$ is dense in $B_{1} \otimes B_{2}$. To see this let ${ }_{n} P_{m}^{i}$ be the ${ }_{n} P_{m}$ defined in $\S 1$ for $\Omega_{i}$, and define

$$
\text { (1) } \begin{gathered}
A_{m}=x \otimes y- \\
=\sum_{k, j=1}^{m}\left\langle x, x_{k}^{\prime}\right\rangle\left\langle y, y_{j}^{\prime}\right\rangle x_{k} \otimes y_{j}=x \otimes y-\left[{ }_{1} P_{m}^{1} x\right] \otimes\left[{ }_{1} P_{m}^{2} y\right] \\
=x \otimes\left[y-{ }_{1} P_{m}^{2} y\right]+\left[x-{ }_{1} P_{m}^{1} x\right] \otimes{ }_{1} P_{m}^{2} y .
\end{gathered}
$$

Then

$$
\gamma\left(A_{m}\right) \leqq\|x\|\left\|y-{ }_{1} P_{m}^{2} y\right\|+\left\|x-{ }_{1} P_{m}^{1} x\right\|\left\|{ }_{1} P_{m}^{2} y\right\| .
$$

The right hand side of (2) tends to zero with $m^{-1}$. This argument extends by linearity to sums of elements of the form $x \otimes y$.

Let now $T_{q}$ be the ${ }_{1} P_{q}$ defined in $\S 1$ corresponding to $\Omega_{1} \otimes \Omega_{2}$. It remains to show that $\left\{T_{q}\right\}_{q}$ is uniformly bounded. It is easy to show that each $T_{q}$ has one of the following three forms: ${ }_{1} P_{n}^{1} \otimes{ }_{1} P_{n}^{2},{ }_{1} P_{n}^{1} \otimes$ ${ }_{1} P_{n}^{2}+{ }_{n+1} P_{n+1}^{1} \otimes{ }_{1} P_{m}^{2},{ }_{1} P_{m}^{1} \otimes_{n+1} P_{n+1}^{2}$. Hence, it suffices to show that $\left.{ }_{n} P_{m}^{1} \otimes_{q} P_{r}^{2}\right\}_{q, r, m}$ is uniformly bounded. Let $M$ be a common bound for all ${ }_{n} P_{m}^{1}$ and ${ }_{q} P_{r}^{2}$. For $\Sigma x \otimes y \in B_{1} \otimes B_{2}$

$$
\begin{gathered}
\gamma\left[{ }_{n} P_{m}^{1} \otimes{ }_{q} P_{r}^{2}(\Sigma x \otimes y)\right]=\gamma\left[\Sigma\left({ }_{n} P_{m}^{1} x\right) \otimes\left({ }_{q} P_{r}^{2} y\right)\right] \\
\leqq(\Sigma\|x\|\|y\|) M^{2} .
\end{gathered}
$$


Since ( 3 ) holds for any representation $\Sigma x \otimes y$ of a given tensor product element, we may replace in it the sum $\Sigma\|x\|\|y\|$ by $\gamma(\Sigma x \otimes y)$, thereby proving our assertion. From Lemma 1, we can conclude that $\Omega_{1} \otimes \Omega_{2}$ defines a basis for $B_{1} \otimes_{\gamma} B_{2}$.

3. The space of completely continuous transformations. We recall that there is a canonical imbedding of $B$, with a biorthogonal set $\Omega$ defining a basis of $B$, into $\left(B^{\Omega}\right)^{\prime}$. The norm of the image of an element $x \in B$ is less than or equal to $\|x\|$. We say that $\Omega$ satisfies the condition of isometry if the imbedding is actually an isometery. For such an $\Omega$, $\left(B^{2}\right)^{\Omega}=B$, isometrically. We state first the following corollary of Theorem 1 .

COROLlaRy 1. If $\Omega_{k}$ is a biorthogonal set defining a basis for $B_{k}, k=1,2$, then $\Omega_{1} \otimes \Omega_{2}$ defines a basis for $B_{1}^{\Omega_{1}} \otimes_{\lambda} B_{2}^{\Omega_{2}}$.

Proof. Each $x_{i}^{\prime} \otimes y_{j}^{\prime}$ is an element of $B_{1}^{\Omega_{1}} \otimes B_{2}^{\Omega_{2}}$ which, as a subset of $B_{1}^{\prime} \otimes_{\lambda} B_{2}^{\prime}$, can be imbedded isometrically in $\left(B_{1} \otimes_{\gamma} B_{2}\right)^{\prime}[4, p .47$, Theorem 3.2]. What is more, the vector space spanned by $\left\{x_{i}^{\prime} \otimes y_{j}^{\prime}\right\}_{i, j}$ is dense, with respect to $\lambda$, in $B_{1}^{\Omega_{1}} \otimes B_{2}^{\Omega_{2}}$, hence in $B_{1}^{\Omega_{1}} \otimes{ }_{\lambda} B_{2}^{\Omega_{2}}$. This is true because

$$
\begin{gathered}
\lambda\left[x^{\prime} \otimes y^{\prime}-\left(\sum_{i=1}^{n}\left\langle x_{i}, x^{\prime}\right\rangle x_{i}^{\prime}\right) \otimes\left(\sum_{i=1}^{n}\left\langle y_{i}, y^{\prime}\right\rangle y_{i}^{\prime}\right)\right] \leqq \gamma\left[x^{\prime} \otimes y^{\prime}-\right. \\
\left.\left(\sum_{i=1}^{n}\left\langle x_{i}, x^{\prime}\right\rangle x_{i}^{\prime}\right) \otimes\left(\sum_{i=1}^{n}\left\langle y_{i}, y^{\prime}\right\rangle y_{i}^{\prime}\right)\right]
\end{gathered}
$$

and the latter quantity tends to 0 . Hence $B_{1}^{\Omega_{1}} \otimes_{\lambda} B_{2}^{\Omega_{2}}=\left(B_{1} \otimes_{\gamma} B_{2}\right)^{\Omega_{1} \otimes \Omega_{2}}$. Our result is a consequence of this.

The next theorem follows easily from this corollary.

THEOREM 2. If both $\Omega_{1}$ and $\Omega_{2}$ satisfy the condition of isometry $\Omega_{1} \otimes \Omega_{2}$ defines a basis for $B_{1} \otimes_{\lambda} B_{2}$.

Proof. If in Corollary 1 we replace $B_{1}$ by $B_{1}^{\Omega_{1}}$ and $B_{2}$ by $B_{2}^{\rho_{2}}$, we conclude that $\Omega_{1} \otimes \Omega_{2}$ defines a basis for $\left(B_{1}^{\Omega_{1}}\right)^{\Omega_{1}} \otimes_{\lambda}\left(B_{2}^{\Omega_{2}}\right)^{\Omega_{2}}$. When the condition of isometry is satisfied the last tensor product can be identified with $B_{1} \otimes_{\lambda} B_{2}$, owing to the relations $B_{k}=\left(B_{k}^{\Omega_{k}}\right)^{\Omega_{k}}$ for $k=1,2$, and the universal character of $\lambda$, [4, p. 35, Lemma 2.12].

Theorem 2 can be considered as a sharpening of the well known fact that if $B_{1}$ and $B_{2}$ have bases, then every completely continuous linear transformation of $B_{1}^{\prime}$ into $B_{2}$ can be uniformly approximated by finite dimensional linear transformations. Our theorem goes further to state that if $\Omega_{1}$ and $\Omega_{2}$ satisfy the condition of isometry, the space of all completely continuous linear transformations of $B_{1}^{\prime}$ into $B_{2}$ has a 
basis consisting of one-dimensional linear transformations.

The condition of isometry deserves some explanation. It is satisfied by a large class of bases, which includes every base for which

$$
B^{\Omega}=B^{\prime}{ }^{(1)}
$$

The equation ( 5 ) holds always for reflexive spaces. It also holds for certain bases of non-reflexive spaces.

A non-reflexive example of (5) is exhibited in [2, p. 188, Example 1], involving the usual basis of $c_{0}, x_{i}=\left\{\delta_{j}^{i}\right\}_{j}$, with $x_{i}^{\prime}=\left\{\delta_{j}^{i}\right\}_{j} \in l^{1}$. An example of the condition of isometry, in the absence (5), is obtained from this first example, by setting [2, p. 188, Example 2] $y_{1}=x_{1}$, and $y_{i}=$ $x_{i}-x_{i-1}+\cdots+(-1)^{i-1} x_{1}$, for $i>1$, and $y_{i}^{\prime}=x_{i}^{\prime}+x_{i+1}^{\prime}$. For $\Omega=\left\{y_{i}, y_{i}^{\prime}\right\}_{i}$, $x_{1}^{\prime} \in B^{\prime} \backslash B^{\Omega} . \quad \Omega$ satisfies the condition of isometry for, if $x \in c_{0}$, then

$$
\left\|\sum_{k=1}^{n}\left\langle x, y_{k}^{\prime}\right\rangle y_{k}\right\|=\left\|\sum_{k=1}^{n}\left\langle x, x_{k}^{\prime}\right\rangle x_{k}\right\| \leqq\|x\| \text {. }
$$

The conclusion is now a consequence of the following theorem and its corollary.

TheOREm 3. If for every $x^{\prime} \in B^{\prime},\left\|_{1} P_{n}^{\prime} x^{\prime}\right\| \rightarrow\left\|x^{\prime}\right\|$, then $\Omega$ satisfies the condition of isometry.

Proof. Let $x_{0} \in B$ and $x_{0}^{\prime} \in B^{\prime}$ such that $\left\|x_{0}^{\prime}\right\|=1$ and $\left\langle x_{0}, x_{0}^{\prime}\right\rangle=\left\|x_{0}\right\|$. Then

$$
\lim _{n \rightarrow \infty} \frac{\left\langle x_{0},{ }_{1} P_{n}^{\prime} x_{0}^{\prime}\right\rangle}{\left\|{ }_{1} P_{n}^{\prime} x_{0}^{\prime}\right\|}=\left\|x_{0}\right\|
$$

CoRollary 2. If $\left\|_{1} P_{n}\right\| \leqq 1$ for every $n$, then $\Omega$ satisfies the condition of isometry.

Proof. We show the above hypothesis implies the hypothesis of Theorem 3. To see this, let $x_{0}^{\prime} \in B^{\prime}$, and $\varepsilon>0$. There is $x_{0} \in B$ so that $\left\|x_{0}\right\|=1$ and $\left.\left\langle x_{0}, x_{0}^{\prime}\right\rangle\right\rangle\left\|x_{0}\right\|-\varepsilon / 2$ and an integer $N>0$ so that

$$
\begin{gathered}
\left\|x_{0}^{\prime}\right\| \geqq\left\|{ }_{1} P_{n}^{\prime} x_{0}^{\prime}\right\| \geqq\left\langle x_{0,1} P_{n}^{\prime} x_{0}^{\prime}\right\rangle=\left\langle{ }_{1} P_{n} x_{0}, x_{0}^{\prime}\right\rangle>\left\langle x_{0}, x_{0}^{\prime}\right\rangle-\varepsilon / 2 \\
>\left\|x_{0}^{\prime}\right\|-\varepsilon,
\end{gathered}
$$

As we have seen, the two biorthogonal sets described above for $c_{0}$ satisfy the hypothesis of Corollary 1.

An example of the isometry condition in which $B^{\prime}$ is not separable is furnished by Schauder's basis for $C([0,1])$, given by the biorthogonal system $\Omega=\left\{x_{i}, x_{i}^{\prime}\right\}_{i}$ described in [1, p. 69]. We consider [0,1] imbedded

1 This equation may be described by saying that $\left\{x_{i}^{\prime}\right\}_{i}$ is a retrobasis for $B^{\prime},[2$, p. 188, Definition 1]. 
in $B^{\prime}$ and treat its points as functionals. The space $B^{\Omega}$ of this example contains the set $D$ of all dyadic fractions. Consequently $\Omega$ satisfies the condition of isometry, since, for $f \in B,\|f\|=\sup _{a \in D}|f(d)|$.

We know of no biorthogonal set defining a basis which does not satisfy the condition of isometry. Neither do we know if $B_{1} \otimes_{\alpha} B_{2}$ has a basis for an arbitrary cross norm $\alpha$, even if $B_{1}$ and $B_{2}$ have bases. It is clear that for any element of $B_{1} \otimes B_{2}$, the formal expansion of Theorem 1 converges to that element with respect to $\alpha$, since it does with respect to $\gamma \geqq \alpha$. The difficulty lies in establishing that the set $\left\{{ }_{p} P_{q}^{1} \otimes{ }_{r} P_{s}^{2}\right\}_{\substack{p, q \\ r, s}}$ is uniformly bounded with respect to $\alpha$.

4. Hilbert spaces and unconditional bases. The problem of approximation of compact operators by finite dimensional operators in a Banach space, can, after elaborate rearrangement, lead to the following question: Can there exist a matrix $C=\left(c_{i j}\right)_{i, j=1}^{\infty}$ satisfying the following conditions:

(a) For some $a_{i} \geqq 0, \sum_{i=1}^{\infty} a_{i}^{2}<\infty,\left|c_{i j}\right| \leqq a_{i} a_{j}$;

(b) $C^{2}=0$;

(c) $\sum_{i=1}^{\infty} c_{i i}=1 ?$

Of course, (b) and (c) are incompatible if $C$ is in the trace class. Thus there arises the question: Does (a) imply that $C$ is in the trace class? To this we can give a definite negative answer via the following theorems.

Therem 4. Let $\Omega=\left\{x_{i}, x_{i}^{\prime}\right\}_{i}, x_{i}=\left\{\delta_{j}^{i}\right\}_{j}, x_{i}^{\prime}=\left\{\delta_{j}^{i}\right\}_{j}$ be the canonical orthonormal basis in $l_{2}$. Then $\Omega \otimes \Omega$ defines an unconditional basis in $l_{2} \otimes_{\gamma} l_{2}$ if and only of condition (a) implies $C$ is in the trace class.

Proof. Let $\Omega \otimes \Omega$ define an unconditional basis for $l_{2} \otimes_{\gamma} l_{2}$. Then we note that (a) may be rephrased by stating: $c_{i j}=\varepsilon_{i j} a_{i} a_{j},\left|\varepsilon_{i j}\right| \leqq 1$. Since $l_{2} \otimes_{\gamma} l_{2}$ is precisely the trace class of operators [4] it follows that $\sum_{i, j=1}^{\infty} \varepsilon_{i j} a_{i} a_{j}\left(x_{i} \otimes x_{j}\right)$ exists in $l_{2} \otimes_{\gamma} l_{2}$ and is therefore in the trace class.

On the other hand, if (a) implies that $C$ is in the trace class, then for $a \otimes a$ in $l_{2} \otimes_{\gamma} l_{2}\left(a=\left(a_{1}, a_{2}, \cdots\right)\right), a \otimes a=\sum_{i, j=1}^{\infty} a_{i} a_{j}\left(x_{i} \otimes x_{j}\right)$. If $B=\left(\varepsilon_{j j} a_{i} a_{j}\right)$ is in the trace class, then $B$ has an expansion $\sum_{i, j=1}^{\infty} \varepsilon_{i j} a_{i} a_{j}$ $\left(x_{i} \otimes x_{j}\right)$, which shows $\Omega \otimes \Omega$ defines an unconditional basis for $l_{2} \otimes_{\gamma} l_{2}$.

Theorem 5. $\Omega \otimes \Omega$ does not define an unconditional basis for $l_{2} \otimes_{\gamma} l_{2}$.

Proof. Let $A_{1}=\left(a_{i j}\right)$ be a $2 \times 2$ matrix with $a_{11}=a_{12}=a_{22}=-a_{21}=1$, and $A_{n}$ the $2^{n} \times 2^{n}$ matrix $\left(A_{i j}\right) i, j=1,2$, with $A_{11}=A_{12}=A_{22}=-A_{21}$ $=A_{n-1}$. Let $B$ be the direct sum of the matrices $\left\{1 / 2^{n / 2} A_{n}\right\}_{n}$. Then a direct computation reveals that $B$ is unitary. Let $B=\left(b_{i j}\right)$, and let 
$C=\left(\left|b_{i j}\right|\right)$. If $\Omega \otimes \Omega$ were an unconditional basis for $l_{2} \otimes_{\gamma} l_{2}$, then for $B$, regarded as a member of $\left(l_{2} \otimes_{\gamma} l_{2}\right)^{\prime}[4$, p. 47, Theorem 3.2] and arbitrary $u \otimes v$ in $l_{2} \otimes_{\gamma} l_{2}, \sum_{i, j=1}^{\infty} u_{i} v_{j}\left\langle x_{i}, B x_{j}\right\rangle$ would converge unconditionally, i.e. $\sum_{i, j=1}^{\infty} u_{k} v_{j}\left|b_{j i}\right|$ would converge. In particular, let $u=v$, where $u$ is given by the vector: $\sum_{n=1}^{\infty}(1 / n) x_{n},\left(\sqrt{2^{n}}\right) x_{n}=\underbrace{(0,0, \cdots 0}$, $\overbrace{1,1, \cdots, 1}^{2^{n}}, 0,0, \cdots)$. A simple verification shows that $u \underbrace{2^{2\left(2 n^{-1}\right.} l_{2}}_{\text {exists in }}$. On the other hand, more calculation shows $\sum_{i, j=1}^{\infty}\left|b_{i j}\right| u_{i} u_{j}=\infty$. The contradiction implies the theorem.

Theorem 5 remains valid when $\gamma$ is replaced by $\lambda$, since $l_{2} \otimes_{\gamma} l_{2}=$ $\left(l_{2} \otimes{ }_{\lambda} l_{2}\right)^{\prime}$, and unconditionality of $\Omega \otimes \Omega$ in $l_{2} \otimes{ }_{\lambda} l_{2}$ implies the same in $l_{2} \otimes_{\gamma} l_{2}$.

Note. We owe to the referee the remark that a space $B$ with a biorthogonal set $\Omega$ which defines a basis for $B$ can always be renormed, preserving the topology of $B$ [1, Theorem 1, p. 67], in such a way that $\Omega$ satisfies the condition of isometry (section 3 ) with respect to the resulting norm of $B$ and the corresponding norm of $B^{\prime}$. This makes. possible the following completely general form of Theorem 2.

THeOREM 2'. If $\Omega_{i}$ defines a basis for $B_{i}$, for $i=1,2$, then $\Omega_{1} \otimes \Omega_{2}$. defines $a$ basis for $B_{1} \otimes_{\lambda} B_{2}$.

Proof. Renorm $B_{1}$ and $B_{2}$ as indicated above. Then, if $\lambda^{\prime}$ denotes the operator norm with respect to the new norms of $B_{1}$ and $B_{2}, B_{1} \otimes_{\lambda^{\prime}} B_{2}$ has a basis defined by $\Omega_{1} \otimes \Omega_{2}$ (Theorem 2). But $B_{1} \otimes_{\lambda^{\prime}} B_{2}=B_{1} \otimes_{\lambda} B_{2}$ : both point-set-wise and topologically. Hence our conclusion.

\section{BIBLIOGRAPHY}

1. M. M. Day, Normed linear spaces, Ergebnisse der Math. und ihre Grenzgebiete, N. F. 28, 1958.

2. B. R. Gelbaum, Expansions in Banach Spaces, Duke Math. J., 17 (1950), 187-196.

3. A. Grothendieck, Produits tensoriels topologiques et espaces nucléaires, Mem. Amer. Math. Soc. No. 16, (1955).

4. R. Schatten, A theory of cross spaces, Ann. Math. Studies. No. 26, Princeton Univ. Press, Princeton 1950.

INSTITUTE FOR ADVANCED STUDY, UNIVERSITY OF MINNESOTA 


\section{PACIFIC JOURNAL OF MATHEMATICS}

\section{EDITORS}

\author{
RaLPh S. Phillips \\ Stanford University \\ Stanford, California \\ F. H. BRowNELL \\ University of Washington \\ Seattle 5 , Washington
}

A. L. Whiteman

University of Southern California

Los Angeles 7, California

L. J. Paige

University of California

Los Angeles 24, California

\author{
E. F. BECKENBACH \\ T. M. CHERRY
}

\author{
ASSOCIATE EDITORS

$\begin{array}{lll}\text { D. DERRY } & \text { H. L. ROYDEN } & \text { E. G. STRAUS } \\ \text { M. OHTSUKA } & \text { E. SPANIER } & \text { F. WOLF }\end{array}$

\section{SUPPORTING INSTITUTIONS}

\author{
UNIVERSITY OF BRITISH COLUMBIA \\ CALIFORNIA INSTITUTE OF TECHNOLOGY \\ UNIVERSITY OF CALIFORNIA \\ MONTANA STATE UNIVERSITY \\ UNIVERSITY OF NEVADA \\ NEW MEXICO STATE UNIVERSITY \\ OREGON STATE COLLEGE \\ UNIVERSITY OF OREGON \\ OSAKA UNIVERSITY \\ UNIVERSITY OF SOUTHERN CALIFORNIA
}

\author{
STANFORD UNIVERSITY \\ UNIVERSITY OF TOKYO \\ UNIVERSITY OF UTAH \\ WASHINGTON STATE COLLEGE \\ UNIVERSITY OF WASHINGTON \\ AMERICAN MATHEMATICAL SOCIETY \\ CALIFORNIA RESEARCH CORPORATION \\ HUGHES AIRCRAFT COMPANY \\ SPACE TECHNOLOGY LABORATORIES \\ NAVAL ORDNANCE TEST STATION
}

Mathematical papers intended for publication in the Pacific Journal of Mathematics should be typewritten (double spaced), and the author should keep a complete copy. Manuscripts may be sent to any one of the four editors. All other communications to the editors should be addressed to the managing editor, L. J. Paige at the University of California, Los Angeles 24, California.

50 reprints per author of each article are furnished free of charge; additional copies may be obtained at cost in multiples of 50 .

The Pacific Journal of Mathematics is published quarterly, in March, June, September, and December. The price per volume (4 numbers) is $\$ 12.00$; single issues, $\$ 3.50$. Back numbers are available. Special price to individual faculty members of supporting institutions and to individual members of the American Mathematical Society: $\$ 4.00$ per volume; single issues, $\$ 1.25$.

Subscriptions, orders for back numbers, and changes of address should be sent to Pacific Journal of Mathematics, 103 Highland Boulevard, Berkeley 8, California.

Printed at Kokusai Bunken Insatsusha (International Academic Printing Co., Ltd.), No. 6, 2-chome, Fujimi-cho, Chiyoda-ku, Tokyo, Japan.

\section{PUBLISHED BY PACIFIC JOURNAL OF MATHEMATICS, A NON-PROFIT CORPORATION}

The Supporting Institutions listed above contribute to the cost of publication of this Journal, but they are not owners or publishers and have no responsibility for its content or policies.

Reprinted 1966 in the United States of America 


\section{Pacific Journal of Mathematics}

\section{Vol. 11, No. 4}

A. V. Balakrishnan, Prediction theory for Markoff processes . . . . . . . . . . 1171

Dallas O. Banks, Upper bounds for the eigenvalues of some vibrating systems . . . . 1183

A. Białynicki-Birula, On the field of rational functions of algebraic groups ...... 1205

Thomas Andrew Brown, Simple paths on convex polyhedra .............. 1211

L. Carlitz, Some congruences for the Bell polynomials . . . . . . . . . . . . 1215

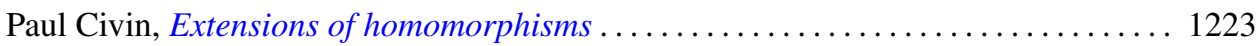

Paul Joseph Cohen and Milton Lees, Asymptotic decay of solutions of differential

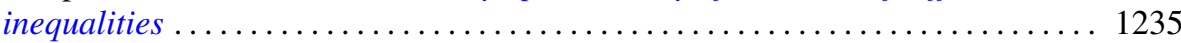

István Fáry, Self-intersection of a sphere on a complex quadric . . . . . . . . . . 1251

Walter Feit and John Griggs Thompson, Groups which have a faithful representation

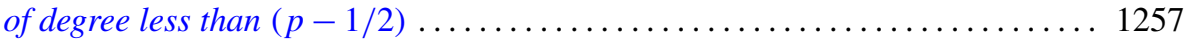

William James Firey, Mean cross-section measures of harmonic means of convex

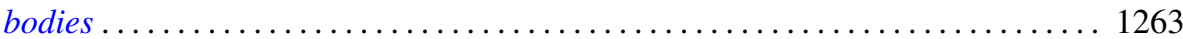

Avner Friedman, The wave equation for differential forms . . . . . . . . . . 1267

Bernard Russel Gelbaum and Jesus Gil De Lamadrid, Bases of tensor products of

Banach spaces ................................... 1281

Ronald Kay Getoor, Infinitely divisible probabilities on the hyperbolic plane . . . . 1287

Basil Gordon, Sequences in groups with distinct partial products . . . . . . . . . . . . 1309

Magnus R. Hestenes, Relative self-adjoint operators in Hilbert space . . . . . . . . . 1315

Fu Cheng Hsiang, On a theorem of Fejér ......................... 1359

John McCormick Irwin and Elbert A. Walker, On N-high subgroups of Abelian

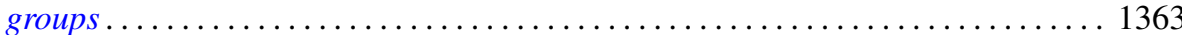

John McCormick Irwin, High subgroups of Abelian torsion groups . . . . . . . . . 1375

R. E. Johnson, Quotient rings of rings with zero singular ideal . . . . . . . . . . . 1385

David G. Kendall and John Leonard Mott, The asymptotic distribution of the time-to-escape for comets strongly bound to the solar system ...

Kurt Kreith, The spectrum of singular self-adjoint elliptic operators ....

Lionello Lombardi, The semicontinuity of the most general integral of the calculus of variations in non-parametric form ................................

Albert W. Marshall and Ingram Olkin, Game theoretic proof that Chebyshev inequalities are sharp

Wallace Smith Martindale, III, Primitive algebras with involution . . William H. Mills, Decomposition of holomorphs ..............

James Donald Monk, On the representation theory for cylindric algebras . . . . . . 1447

Shu-Teh Chen Moy, A note on generalizations of Shannon-McMillan theorem . . . . 1459

Donald Earl Myers, An imbedding space for Schwartz distributions . .

John R. Myhill, Category methods in recursion theory .........

Paul Adrian Nickel, On extremal properties for annular radial and circular slit mappings of bordered Riemann surfaces

Edward Scott O'Keefe, Primal clusters of two-element algebras . .

Nelson Onuchic, Applications of the topological method of Wazewski to certain

problems of asymptotic behavior in ordinary differential equations ...

Peter Perkins, A theorem on regular matrices................

Clinton M. Petty, Centroid surfaces .... 